\title{
Cas atypique d'uvéite associée à l'antigène HLA-B27 avec hypopyon et atteinte du segment postérieur
}

\author{
PAR THOMAS XIE, OD \& ETTY BITTON, OD, MSc, FAAO
}

\section{RÉSUMÉ}

La présence d'un hypopyon et l'atteinte du segment postérieur sont des signes cliniques peu fréquents dans les cas d'uvéite associée à l'antigène HLA-B27. De plus, les premières attaques sont rares chez les personnes âgées. Ce rapport décrit un cas atypique d'uvéite chez une personne âgée qui s'est présentée à la fois avec un hypopyon visible et une uvéite intermédiaire sévère dans un œil.

Un homme caucasien de 60 ans a consulté à cause d'un œil rouge et douloureux avec baisse subite de la vision au niveau de la perception de la main dans l'œil atteint. Ses antécédents oculaires et systémiques ne présentaient rien de remarquable. Un examen de la chambre antérieure de l'œil a révélé la présence de cellules inflammatoires importantes et un hypopyon flagrant. Un examen du segment postérieur a révélé la présence d'une vaste hyalite, entravant toute visibilité de la rétine. En dépit de l'âge et des signes oculaires atypiques du patient, un diagnostic d'une uvéite liée à l'antigène HLA-B27 à la suite d'une évaluation approfondie des signes cliniques et des résultats d'analyses de laboratoire a été proposé. Linflammation a été traitée avec succès au moyen d'une combinaison de corticostéroïdes administrés par voies intraveineuse, topique et orale dont la dose a diminué progressivement en quelques semaines. L'acuité visuelle s'est rétablie à 20/30.

Ce cas constitue un rappel important du fait que des signes atypiques comme un hypopyon ou une uvéite intermédiaire peuvent apparaître et constituer un signe important d'uvéite liée à l'antigène HLA-B27. Les cliniciens doivent connaître les différentes manifestations de l'uvéite associée à l'antigène HLA-B27 et agir avec prudence en incluant une évaluation complète des segments antérieur et postérieur face à un œil rouge et douloureux associé à une baisse de vision.

Mots clés : uvéite antérieure, uvéite intermédiaire, HLA-B27, hypopyon

\section{Introduction}

T 'uvéite, la forme la plus cou1 rante d'inflammation oculaire, constitue une importante préoccupation en santé publique. Elle cause un pourcentage important (estimé de 10 à $15 \%$ ) des cas prévalents de cécité légale aux États-Unis. ${ }^{1}$ Le sous-type le plus répandu est l'uvéite antérieure, qui représente jusqu'à $92 \%$ du total des cas dans les pratiques ophtalmiques communautaires ${ }^{2}$. La positivité au HLA (antigène leucocytaire humain) - B27, complexe majeur d'histocompatibilité $(\mathrm{CMH})$ des humains, constitue la cause identifiée la plus courante de l'uvéite antérieure et cause environ $50 \%$ des cas dans des populations différentes. ${ }^{3,4}$ L'uvéite associée au HLA-B27 est caractérisée par des poussées unilatérales aiguës en alternance et récidivantes d'inflammation intraoculaire du segment antérieur de l'œil et touche habituellement les jeunes hommes adultes. ${ }^{5}$ Dans les cas d'uvéite reliée au HLA-B27, la présence d'un hypopyon - amas de globules blanches dans la chambre antérieure - et l'atteinte du segment postérieur de l'œil ne sont pas courantes.

Un hypopyon indique la présence d'une inflammation intraoculaire sévère du segment antérieur et il est rare chez les patients qui ont une uvéite, faisant son apparition dans moins de $1 \%$ des cas. ${ }^{6}$ L'atteinte du segment postérieur, appelée uvéite intermédiaire ou postérieure, est peu fréquente elle aussi et elle varie de 0 à $25 \%$ des cas d'uvéite associée au HLA-B27.-10 De plus, les cas d'uvéite antérieure et intermédiaire présentent un risque plus faible d'hypopyon comparativement aux patients qui ont seulement une uvéite antérieure. ${ }^{6}$

Le présent rapport décrit un cas atypique d'uvéite associée au HLA-B27 chez un homme âgé qui avait à la fois un hypopyon et une uvéite intermédiaire sévère.

\section{Rapport de cas}

Un homme caucasien de 60 ans s'est présenté à la clinique oculaire d'un hôpital en se plaignant d'avoir l'œil droit douloureux, rouge, avec une baisse de la vision. Le patient avait en fait été vu trois jours plus tôt à cause d'une douleur et d'une inflammation causées par un traumatisme à l'épaule droite pour lesquelles on lui avait prescrit un analgésique narcotique (oxycodone $5 \mathrm{mg}$ et acétaminophène $325 \mathrm{mg}$ vendu sous la marque Percocet, un comprimé aux quatre heures au besoin). Les symptômes oculaires sont apparus lorsqu'il a commencé à prendre le Percocet, et c'est pourquoi il a cessé de prendre le médicament après une journée, mais sa vision a continué 
Tableau 1 : Présentation clinique initiale

\begin{tabular}{|c|c|c|}
\hline & CEIL DROIT (OD) & CEIL GAUCHE (OS) \\
\hline Acuité visuelle & Mouvement de la main & $20 / 30$ \\
\hline Réflèxes des pupilles & Normaux & Normaux \\
\hline $\begin{array}{l}\text { Mouvements } \\
\text { extraoculaires }\end{array}$ & Normaux & Normaux \\
\hline Tonométrie (TonoPèn) & $21 \mathrm{mmHg}$ & $22 \mathrm{mmHg}$ \\
\hline Conjonctive bulbaire & Injection 2+ & \multirow{5}{*}{ Rien de remarquable } \\
\hline Cornée & Précipités kératiques fins généralisés & \\
\hline Chambre antérieure & $\begin{array}{l}\text { Cellules } 4+\text { et « flare », production de } \\
\text { fibrines et hypopyon de 1,5 mm de } \\
\text { hauteur, angle Van Herick grade IV }\end{array}$ & \\
\hline Cristallin & $\begin{array}{l}\text { Sclérose nucléaire 1+ sans synéchies } \\
\text { postérieures }\end{array}$ & \\
\hline Segment postérieur & $\begin{array}{l}\text { Cellules et débris inflammatoires vitréens } \\
\text { prononcés (grade } 4+\text { ) }\end{array}$ & \\
\hline
\end{tabular}

à se détériorer. Ses antécédents oculaires ne présentaient rien de remarquable et ne comportaient aucun traumatisme, ni chirurgie, inflammation ou infection. Comme médicaments, il prenait seulement le Percocet pour sa douleur à l'épaule depuis peu et, à l'occasion, un anti-inflammatoire non stéroïdien (naproxen) pour une douleur non spécifique dans le corps. Il n'avait aucune allergie déclarée à aucun médicament. L'examen de tous les systèmes a révélé des poussées appelées podagre (c.-à-d. inflammation du gros orteil liée à des récidives de goutte $)^{11}$ au cours de la dernière année (même si l'on n'a jamais diagnostiqué officiellement qu'il avait la goutte) et des antécédents d'éruptions papulaires au front et aux deux tibias avec érythème bénin et un peu d'excoriation. Suite à un questionnement plus approfondi, le patient n'a signalé aucun antécédent d'ulcère, de lésion, de syndrome du côlon irritable, de selles sanguinolentes, de difficulté à uriner ou d'essoufflement. Les antécédents familiaux du patient ne présentaient rien de remarquable. Le patient a aussi affirmé ne pas abuser de la nicotine et de l'alcool et ne pas avoir eu de rapport sexuel récent. Le patient avait conscience du temps, de l'espace et de sa personne, et il était lucide au moment de l'examen.

L'examen oculaire a révélé que l'acuité visuelle (AV) était limitée au mouvement de la main OD (sans amélioration au trou sténopéique) et s'établissait à 20/30 OS. L'examen biomicroscopique de l'œil droit a révélé une injection $2+$ de la conjonctive bulbaire. Des précipités kératiques fins étaient distribués généralement sur toute la cornée. Aucun amincissement n'a été constaté. L'examen de la chambre antérieure (CA) de l'œil

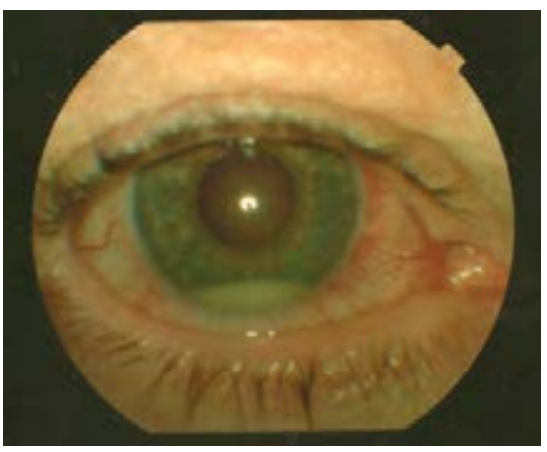

Figure 1 - Hypopyon (hauteur de $1,5 \mathrm{~mm}$ ) dans le quadrant inférieur de la chambre antérieure.

droit a révélé la présence de cellules $4+$ et un effet Tyndall (sans courant de convection), la production de fibrine et un hypopyon d'une hauteur de $1,5 \mathrm{~mm}$ dans le quadrant inférieur comme le montre la Figure 1. L'examen du fond d'oeil avec dilatation pupillaire a révélé la présence d'un brouillard sévère du vitré (cellules inflammatoires grade 4+) bloquant la vue de la rétine. Une ultrasonographie de l'oeil (B-Scan) a révélé la présence de nombreux débris au vitré. L'examen de l'œil gauche a révélé seulement la présence d'une sclérose nucléaire précoce sans signe d'inflammation active ou antérieure. Le Tablean 1 résume les observations cliniques.

Après l'échographie $\mathrm{B}$, on a diagnostiqué chez le patient une uvéite à hypopyon antérieure et intermédiaire non granulomateuse unilatérale et aiguë en lien présumé avec l'anticorps HLA-B27. Le patient a reçu par voie intraveineuse $125 \mathrm{mg}$ de glucocorticoïde anti-inflammatoire (succinate sodique de méthylprednisolone) jumelé à un corticostéroïde antiinflammatoire topique (acétate de 
Tableau 2 : Résultats de laboratoire cliniques

\begin{tabular}{|c|c|c|c|}
\hline & OBJET & VALEURS NORMALES & RÉSULTAT \\
\hline $\begin{array}{l}\text { Taux de } \\
\text { sédimentation }\end{array}$ & Inflammation & $\leq 30 \mathrm{~mm} / \mathrm{h}$ & $\begin{array}{l}\text { Élévation } \\
(110 \mathrm{~mm} / \mathrm{h})\end{array}$ \\
\hline Protéine C-réactive & Inflammation & $<6 \mathrm{mg} / \mathrm{L}$ & $\begin{array}{l}\text { Élévation } \\
(19,35 \mathrm{mg} / \mathrm{L})\end{array}$ \\
\hline Globules blanches & Inflammation & $4 \times 10^{9}$ à $1,1 \times 10^{10} / \mathrm{L}$ & $\begin{array}{l}\text { Légère leucocytose } \\
\left(13,1 \times 10^{9} / L\right)\end{array}$ \\
\hline $\begin{array}{l}\text { Antigène leucocytaire } \\
\text { humain B27 (HLA-B27) }\end{array}$ & $\begin{array}{l}\text { Protéine spécifique } \\
\text { fortement liée aux } \\
\text { spondyloarthropathies }\end{array}$ & Négatif & Positif \\
\hline $\begin{array}{l}\text { IgG du virus de } \\
\text { l'herpès simplex }\end{array}$ & $\begin{array}{l}\text { Anticorps spécifiques du } \\
\text { virus de l'herpès simplex }\end{array}$ & Négatif & Positif \\
\hline $\begin{array}{l}\text { IgM du virus de } \\
\text { l'herpès simplex }\end{array}$ & $\begin{array}{l}\text { Anticorps spécifiques du } \\
\text { virus de l'herpès simplex }\end{array}$ & Négatif & Positif \\
\hline $\begin{array}{l}\text { Enzyme de conversion } \\
\text { de l'angiotensine }\end{array}$ & Sarcoïdose & Négatif & Négatif \\
\hline Toxoplasma & Toxoplasmose & Négatif & Négatif \\
\hline $\begin{array}{l}\text { Anticorps du } \\
\text { Treponema pallidum } \\
\text { (FTA-ABS) }\end{array}$ & Syphilis & Négatif & Négatif \\
\hline $\begin{array}{l}\text { Anticorps réaginique } \\
\text { (RPR) }\end{array}$ & Syphilis & Négatif & Négatif \\
\hline $\begin{array}{l}\text { Dosage du virus zona } \\
\text { varicelle (VZV) }\end{array}$ & $\begin{array}{l}\text { Anticorps du virus } \\
\text { zona varicelle }\end{array}$ & Négatif & Négatif \\
\hline
\end{tabular}

prednisolone à $1 \%$ qh, avec dose d'attaque avant le coucher et au réveil), un agent cycloplégique/ mydriatique (atropine à $1 \%$ tid) et un corticostéroïde anti-inflammatoire à prendre par voie orale (prednisone $80 \mathrm{mg}$ tous les jours). Le patient a ensuite été envoyé au laboratoire pour une prise de sang devant servir à une analyse plus poussée.

Une étude subséquente des résultats du bilan sanguin (Tableau 2) a révélé une élévation de taux de sédimentation, protéine C-réactif et des globules blancs. Les résultats de laboratoire étaient positifs pour les marqueurs suivants : HLA-B27, IgG et IgM du virus de l'Herpès. Les autres résultats de l'analyse, y compris ACE, Toxoplasma, FTA-ABS, RPR et titrage VZV, étaient négatifs. L'étiologie de l'uvéite a donc été confirmée comme positive au HLA-B27. Le patient n’avait pas de médécin généraliste et c'est pourquoi on a recommandé une consultation avec un rheumatologue.

Le patient a bien répondu le début du traitement, l'acuité visuelle de l'œil droit s'était améliorée à 20/60, la PIO s'établissait à $16 \mathrm{mmHg}$, le segment antérieur a révélé la présence de quelques précipités kératiques dans la partie inférieure, des cellules $1+$ et des cellules inflammatoires dans la au traitement. Dix jours après
CA, de même qu'un hypopyon de $<0,5 \mathrm{~mm}$. Un examen du fond de l'œil avec dilatation pupillaire a démontré la présence de cellules vitréennes antérieures $1+$ et des accumulations d'exsudats étendues ("snowbanks») et limitées (" snowballs») à la partie inférieure. La macula, le nerf optique et le reste de la rétine ne présentaient rien de remarquable. À cause de l'amélioration subjective et objective prononcées, la dose de prednisone a était progressivement réduite $(60 \mathrm{mg} \times 5$ jours, $40 \mathrm{mg} \times 5$ jrs, $30 \mathrm{mg} \times 5$ jrs, $20 \mathrm{mg} \times 5$ jrs, 10 $\mathrm{mg} \times 5 \mathrm{jrs}, 5 \mathrm{mg} \times 5 \mathrm{jrs}$ et ensuite un arret du médicament).

Au cours de la troisième semaine, l'évolution clinique était favorable et l'AV s'établissait à $20 / 30^{-2}$. Les précipités et l'hypopyon ont disparu (Figure 2), mais des cellules inflammatoires dans la CA de grade 0,5+ sont demeurés, ainsi que des cellules au vitré antérieur de $0.5+$ et une persistance d'exsudat à la rétine. L'œil gauche est demeuré stable et sans manifestation durant l'épisode. Au dernier suivi à neuf semaines, le patient avait déjà cessé de prendre les médicaments systémiques et topiques une semaine plus tôt. Son AV s'était maintenue à $20 / 30^{-1}$, la PIO était de $16 \mathrm{mmHg}$, le segment antérieur ne présentait rien de remarquable et un examen du segment postérieur a révélé la présence de cellules vitréennes de grade $0,5+$ et d'exsudats limités à la partie inférieure, entre 5 à 8 heures. Le patient devait revenir pour un suivi périodique un mois plus tard. 
Tableau 3 : Étiologies possibles de l'uvéite

\begin{tabular}{|c|c|c|}
\hline & DESCRIPTION GÉNÉRALE & MANIFESTATIONS OCULAIRES \\
\hline Positivité au HLA-B275 & $\begin{array}{l}\text { Marqueur génétique associé aux spondyloarthropathies } \\
\text { Cause identifiable la plus courante de l'uvéite } \\
\text { antérieure (50\%) }\end{array}$ & Uvéite antérieure \\
\hline Uvéite à herpès simplex $x^{12,13,35-38}$ & $\begin{array}{l}\text { Uvéite causée par le virus de l'herpès simplex } \\
\text { Secondaire à une kératite herpétique } \\
\text { Aucune atteinte cornéenne dans } 15 \% \text { des cas }\end{array}$ & $\begin{array}{l}\text { Cicatrisation cornéenne } \\
\text { Uvéite antérieure récidivante unilatérale } \\
\text { Atrophie de l'iris, élévation de la PIO, précipités et synéchies } \\
\text { Hypopyon possible } \\
\text { Inflammation du segment postérieur dans } 15 \% \text { des cas }\end{array}$ \\
\hline Maladie de Behçet ${ }^{14}$ & $\begin{array}{l}\text { Vasculite systémique d'étiologie inconnue } \\
\text { Récidive d'ulcères dans la bouche, d'ulcères génitaux } \\
\text { et de lésions cutanées }\end{array}$ & Crise subite d'uvéite antérieure avec hypopyon \\
\hline Sarcoïdose ${ }^{15,16,17}$ & $\begin{array}{l}\text { Maladie chronique systémique granulomateuse sans } \\
\text { caséification d'étiologie inconnue } \\
\text { Atteinte oculaire chez le tiers des patients }\end{array}$ & $\begin{array}{l}\text { Uvéite antérieure bilatérale chronique } \\
\text { Nodules iridiens, synéchies postérieures, précipités } \\
\text { et synéchies antérieures périphériques } \\
\text { Atteinte possible du segment postérieur }\end{array}$ \\
\hline Toxoplasmose $2,18,19$ & $\begin{array}{l}\text { Infection causée par le parasite Toxoplasma gondii } \\
\text { Cause infectieuse la plus courante d'inflammation } \\
\text { intraoculaire chez les patients immunocompétents }\end{array}$ & Peut causer l'uvéite antérieure et la vasculite rétinienne \\
\hline $\begin{array}{l}\text { Ophtalmicus à herpès zoster } \\
\text { (zona oculaire })^{20}\end{array}$ & $\begin{array}{l}\text { Réactivation du virus zona de la varicelle } \\
\text { Complications oculaires chez } 50 \% \text { des patients } \\
\text { avec uvéite dans } 43 \% \text { des cas }\end{array}$ & $\begin{array}{l}\text { Incidence élevée de glaucome secondaire } \\
\text { Uvéite à phase unique et de brève durée }\end{array}$ \\
\hline Syphilis ${ }^{21-24}$ & $\begin{array}{l}\text { Infection à Treponema pallidum transmise sexuellement } \\
\text { Plusieurs personnes n'ont pas de symptômes } \\
\text { Peut se manifester au début sous forme d'uvéite antérieure }\end{array}$ & $\begin{array}{l}\text { Peut imiter de nombreuses conditions oculaires. } \\
\text { L'uvéite peut être unilatérale ou bilatérale, aiguë } \\
\text { ou chronique } \\
\text { Peut causer l'apparition d'un hypopyon }\end{array}$ \\
\hline Uvéite tuberculeuse ${ }^{25}$ & $\begin{array}{l}\text { Rare } \\
\text { Causée par Mycobacterium tuberculosis } \\
2 \% \text { des cas de tuberculose active }\end{array}$ & $\begin{array}{l}\text { Uvéite antérieure chronique granulomateuse } \\
\text { Choroïdite disséminée avec vitrite } \\
\text { Edème maculaire kystoïde }\end{array}$ \\
\hline
\end{tabular}



Figure 2: Photo du segment antérieur illustrant l'bypopyon résorbé et une chambre antérieure claire.

\section{Discussion}

Le diagnostic différentiel dans le cas de ce patient a inclus des étiologies infectieuses et non infectieuses de l'uvéite, ce qui comprend la positivité à l'antigène HLA-B27, le virus de l'herpès simplex, la maladie de Behçet, la sarcoïdose, la toxoplasmose, le virus zona de la varicelle (VZV), la syphilis et la tuberculose. Même si elles sont moins probables, la sclérose en plaques, la maladie de Lyme et Bartonella auraient aussi pu faire partie de la liste des diagnostics différentiels. Le Tablean 3 présente une brève description de chaque étiologie.

Le patient a été diagnostiqué initialement avec une uvéite nongranulomateuse liée tentativement au HLA-B27, mais l'hypopyon et l'uvéite intermédiaire étaient atypiques. Ramsay et Lightman (2001) ont classifié les causes de l'hypopyon en causes non infectieuses, agents infectieux, néoplasmes et troubles de la cornée. ${ }^{26}$ Le Tableau 4 présente le diagnostic différentiel le plus courant de l'hypopyon, de l'uvéite antérieure et de l'uvéite intermédiaire. 
Tableau 4 : Diagnostic différentiel le plus courant d'hypopyon, d'uvéite antérieure et d'uvéite intermédiaire

\begin{tabular}{|l|l|l|}
\hline \multicolumn{1}{|c|}{ HYPOPYON } & \multicolumn{1}{c|}{ UVÉITE ANTÉRIEURE } & \multicolumn{1}{c|}{ UVÉITE INTERMÉDIAIRE } \\
\hline Non infectieux & Idiopathique & Idiopathique \\
HLA-B27 & SLA-B27 & Scérose en plaques \\
Maladie de Behçet & Sarcoïdose \\
Spondyloarthropathie & Arthrite réactionnelle (Reiter) & Maladie de Lyme \\
latrogène/Néoplasme/Traumatisme & Spondylite ankylosante & \\
- Sarcoïdose & \\
Infectieux & Syphilis & \\
Endophtalmite endogène & Virus de l'herpès simplex & \\
Toxoplasmose & Traumatisme \\
Syphilis & Syndrome de Posner-Schlossman & \\
Maladie de Hansen & Néoplasme \\
Brucellose & Lymphome, leucémie, rétinoblastome \\
Virus de l'herpès simplex & Autre \\
Kératite & \\
Autre & & \\
\hline
\end{tabular}

Dans les cas d'infections et d'inflammations intraoculaires, l'hypopyon est constitué en grande partie de débris de tissus, de fibrine, de sous-produits de l'inflammation et de leucocytes, et signifie qu'il y a une inflammation intraoculaire sévère du segment antérieur. ${ }^{6}$ Une étude de Zaidi et al indique que la présence d'hypopyon est peu courante dans les cas d'uvéite, représentant seulement 8,57 patients qui ont une uvéite et fait son apparition chez quelque 8,57 patients par 1000 années-personne $(0,86 \%)$ même dans les pratiques de soins tertiaires de l'uvéite. Cette étude rétrospective a indiqué que l'hypopyon était plus courant chez les patients atteints d'une uvéite limitée à la chambre antérieure que chez ceux qui avaient également une uvéite intermédiaire diagnostiquée, mais presque aussi fréquent chez les patients qui avaient une uvéite postérieure ou une panuvéite. Les facteurs de risque les plus courants d'hypopyon sont la maladie de Behçet et la positivité au HLA-B27, ce qui quintuple et double environ le risque d'hypopyon respectivement. En fait, l'uvéite à hypopyon liée au HLA-B27 se manifeste dans $5,7 \%$ de tous les cas d'uvéite et est plus courante chez les personnes caucasiennes. ${ }^{27}$ Même si l'hypopyon est un indicateur d'une inflammation sévère, les yeux qui le développent ne sont pas plus à risque pour des troubles visuels que ceux qui n'en développent pas. Une étude antérieure portant sur la maladie de Behçet a montré que les patients chez lesquels un hypopyon faisait son apparition étaient plus susceptibles de regagner trois lignes d'acuité visuelle lors du suivi, probablement parce que l'embrouillement associé à l'hypopyon constituait une cause réversible de perte de vision. ${ }^{28}$

On diagnostique une uvéite intermédiaire lorsque l'inflammation intraoculaire atteint principalement le vitré, la région périphérique de la rétine et la pars plana ciliaris. ${ }^{29}$ Il s'agit du type d'uvéite dont le suivi de rétablissement est le plus long. ${ }^{30}$ Le syndrome est plus fréquent au cours de la troisième et de la quatrième décennie. ${ }^{31} \mathrm{On}$ a signalé que l'uvéite intermédiaire constitue de 1,4 à $22 \%$ de tous les cas d'uvéite. ${ }^{32}$ Même si la majorité des cas sont d'étiologie inconnue, on a signalé un lien important entre l'uvéite intermédiaire et la sclérose en plaques, la sarcoïdose et la maladie de Lyme. ${ }^{33,34}$ L'uvéite intermédiaire est bilatérale dans $80 \%$ des cas. Les principales caractéristiques cliniques sont les cellules de vitré, avec ou sans accumulation limitée ou étendue d'exsudats. L'uvéite intermédiaire constatée dans le cas décrit était atypique, car elle était monoculaire et ne présentait aucun lien avec les maladies susmentionnées.

Dans le rapport de cas décrit ici, les résultats de laboratoire du patient étaient positifs pour le HLA-B27, IgG et IgM du virus de l'herpès, ce qui indiquait une étiologie d'uvéite associée au HLA-B27 ou d'uvéite liée à l'herpès simplex.

L’uvéite aigüe liée au virus de l'herpès est habituellement secondaire à la kératite herpétique, par contre il se peut que $15 \%$ des patients n'ont pas d'atteinte cornéenne. ${ }^{35,39}$ 
Les signes cliniques d'uvéite au virus de l'herpès comprennent les cicatrices cornéennes, l'atrophie de l'iris focalisée ou en région, les défauts de transillumination de l'iris, la présence de précipités, de synéchies postérieures, et une hausse de la PIO. ${ }^{39-41}$ Une uvéite antérieure unilatérale combinée à une hausse de $\mathrm{PIO}$ est indicative d'une uvéite reliée au virus de l'herpès. Une atrophie de l'iris ainsi qu'une transillumination raffirme le diagnostic. Dans le cas décrit plus haut, ni l'iris ni la PIO étaient affectés, alors un diagnostic d'uvéite reliée à l'herpès n'était pas retenu et celui relié a une positivité de HLA-B27 était favorisé comme étiologie.

En dépit d'une présentation atypique, le pronostic était positif dans le cas de ce patient, comme dans celui de la plupart des uvéites associées au HLAB27. L'administration de stéroïdes topiques et systémiques a permis une évolution clinique favorable et le patient a retrouvé son AV à $20 / 30^{-1}$ en moins de neuf semaines. Le patient avait une inflammation de l'épaule droite immédiatement avant l'apparition de son uvéite. On ne sait pas trop pour le moment si l'inflammation de l'épaule et l'uvéite constituent deux inflammations distinctes ou si elles font partie d'un seul problème systémique. Les patients qui ont une uvéite antérieure associée au HLA-B27 et des symptômes simultanés au segment postérieur présentent une incidence beaucoup plus élevée de maladies systémiques connexes, comme la spondylite ankylosante, les maladies intestinales inflammatoires ou l'arthrite réactionnelle (auparavant appelée syndrome de Reiter). ${ }^{42}$ On a donc recommandé de référer le patient en rheumatologie pour exclure ces possibilités. On l'a informé que des récidives sont probables et que le processus peut faire partie d'un problème inflammatoire systémique qui peut l'obliger à prendre des stérö̈des ou à suivre une thérapie immunosuppressive chronique. Le patient n'a toutefois pas été vu au cours d'un suivi après la référence.

\section{Uvéite associée au HLA-B27}

Comme diagnostic d'uvéite spécifique le plus répandu, l'uvéite associée au HLA-B27 représente environ 13 à $17 \%$ de tous les cas d'uvéite., 43 Environ $50 \%$ des patients qui ont une uvéite antérieure aigüe sont positifs pour le HLA-B275. L'uvéite associée au HLA-B27 est trois fois plus fréquente chez les hommes. ${ }^{44} \mathrm{La}$ maladie fait son apparition vers l'âge de 35 ans en moyenne, même l'on en a signalé des cas chez des enfants (10\% des cas se manifestent avant l'âge de 20 ans) et chez des adultes d'âge mûr $\left(5 \%\right.$ après 55 ans)..$^{10}$

L'uvéite antérieure aigüe (UAA) constitue la manifestation classique d'une maladie oculaire associée au HLA-B27. ${ }^{10}$ L'apparition est habituellement subite et les symptômes comprennent la photophobie, la douleur oculaire, l'épiphorie, la rougeur oculaire et des troubles de vision variés. Les cas sont en général unilatéraux, mais une récidive peut atteindre l'œil contralatérale. Même si l'inflammation est habituellement non granulomateuse, elle peut être assez sévère pour causer l'apparition d'un hypopyon ou rendre l'humeur aqueuse plasmoïde. L'UAA associée au HLA-B27 est en fait la cause la plus courante d'uvéite à hypopyon en Amérique du Nord. ${ }^{27}$ Dans les cas peu courants (mois que 25,1\%), le segment postérieur peut être atteint. ${ }^{7}$ Cette atteinte du segment postérieur peut inclure une vitrite, un œdème maculaire cystoïde, une papillite, une vasculite rétinienne et l'on croit qu'elle est secondaire à une inflammation du segment antérieur. ${ }^{10,45}$ C'est pourquoi l'uvéite associée au HLA-B27 peut être d'une gravité inhabituelle et provoquer une panuvéite, un phénomène sous-évalué. ${ }^{46} \mathrm{Au}$ cours d'une attaque aigüe, la PIO peut être diminuée à cause d'une baisse de production de l'humeur aqueuse du corps ciliaire.

L'augmentation de la PIO et le glaucome secondaire constituent néanmoins des complications bien reconnues à cause de la présence d'un iris bombé ou la fermeture de l'angle relié aux synéchies. Les crises d'uvéite sont en général de courte durée et disparaissent dans les trois mois. Les récidives sont courantes, mais la fréquence varie entre de multiples crises par année et des crises périodiques à une décennie ou plus d'intervalle. ${ }^{46}$

Le traitement de l'uvéite est graduel. ${ }^{47} \mathrm{Il}$ vise dans l'immédiat à contrôler l'inflammation et les spasmes ciliaires et, à long terme, à s'adresser à la cause sous-jacente de l'uvéite. Il est possible de traiter les premières occurrences et les récidives sans complication au moyen de corticostéroïdes topiques comme l'acétate de predni-solone à $1 \%$, de teneur modérée, qui convient à de nombreux cas. Même si le risque d'effets secondaires systémiques est faible, il est possible de retrouver une 
augmentation de la PIO chez 1\% des patients. On peut alors utiliser une formulation plus douce comme la rimexolone et le lotoprednol. ${ }^{48}$ L'utilisation simultanée d'un cycloplégique/mydriatique comme le sulfate d'atropine à $1 \%$ atténue la douleur causée par le spasme ciliaire et peut dissocier les synéchies postérieures ou en prévenir l'apparition.

Les cas plus sévères peuvent obliger à combiner les traitements oraux, topiques, périoculaires ou intravitréens. Dans 24\% des patients, il faut administrer un corticostéroïde systémique. Une dose de départ habituellement administrée par voie orale est de $1 \mathrm{mg} / \mathrm{kg}$ de prednisone à chaque jour. Les effets secondaires découlant d'un bref traitement aux stéroïdes systémiques sont peu fréquents mais comprennent les troubles du sommeil, la prise de poids, l'augmentation de l'appétit, des déséquilibres de comportement et autres. ${ }^{49}$ Dans les cas extrêmes d'uvéite atteignant le segment postérieur, on peut envisager d'administrer des corticostéroïdes périoculaires ou intravitréens. ${ }^{50}$ Les injections périoculaires (par voie transseptale, dans l'espace sous-tenonien ou par voie sous-conjonctivale) sont conçues comme des injections à effet prolongé (p. ex., acétate de triamcinolone) et sont donc efficaces pendant une période plus longue. ${ }^{51}$ Les injections intravitréennes sont efficaces pendant trois à six mois, réduisent au minimum les effets secondaires systémiques et peuvent traiter l'œdème maculaire causé par l'uvéite postérieure. ${ }^{52}$ Par contre, les complications oculaires (p. ex., cataractes et augmentation de la PIO) sont plus courantes avec les injections intravitréennes qu'avec les stérö̈des systémiques.

Des cas extrêmes qui mettent la vue en danger peuvent nécessiter une intervention intermittente de stéroïdes par voie intraveineuse (IV) afin de contrôler l'inflammation plus rapidement et prévenir des dommages irréparables. Le traitement recommandé dans de tels cas est la méthylprednisolone $1 \mathrm{~g}$ IV par jour pendant trois jours. On passe ensuite à une thérapie orale commençant à $1 \mathrm{mg} / \mathrm{kg}$ par jour. ${ }^{47,53,54}$ Dans le cas des infections récalcitrantes, on peut envisager des stratégies sans stéroïdes comme une thérapie aux AINS (anti-inflammatoire nonstéroïdien) par voie orale ou une thérapie immunosuppressive. ${ }^{55,56,47}$

Les patients qui prennent des stéroïdes topiques ou systémiques pendant une période prolongée (c.-à-d. pendant plus de deux semaines) doivent être sevrés sur plusieurs semaines de façon à éviter l'inflammation de rebond après l'usage topique, ou pour éviter de provoquer une crise surrénale à cause de l'arrêt brutal de l'usage de corticostéroïdes oraux. Les patients qui ont besoin d'une thérapie immunosuppressive devraient suivre leur traitement sans réduire la dose afin d'éviter toute récidive, qui peut être difficile à contrôler.

L'évaluation d'une présence du facteur de HLA-B27 chez les patients souffrant d'uvéite peut être utile, afin d'identifier les cas reliés à des maladies systémiques. Environ $70 \%$ des patients qui ont une uvéite associée au HLA-B27 auront une spondyloarthropathie séronégative (facteur rhumatoïde) associée et l'on n'aura pas posé de diagnostic chez environ $50 \%$ d'entre eux, ou le diagnostic aurait été erroné. La spondyloarthropathie négative inclut la spondylite ankylosante, l'arthrite réactionnelle (syndrome de Reiter), le rheumatisme psoriasique et l'arthrite associée à la maladie intestinale inflammatoire. L'incidence de ces maladies systémiques est beaucoup plus élevée chez les patients qui ont des manifestations au segment postérieur. ${ }^{42,57}$ En outre, de 30 à $90 \%$ des patients qui ont une uvéite associée au HLA-B27 ont aussi une maladie articulaire associée. ${ }^{58}$ Par conséquence, il est important d'évaluer la présence du facteur HLA-B27 afin de pouvoir améliorer la prise en charge globale du patient.

\section{Résumé}

Dans ce rapport de cas, on a posé un diagnostic final d'uvéite associée au HLA-B27 après une évaluation clinique et des analyses de laboratoire détaillées en dépit de la manifestation atypique. L'hypopyon et l'uvéite antérieure et intermédiaire associée ont été traités avec succès au moyen de stéroïdes topiques et systémiques. Ce cas constitue un rappel important : même s'ils sont peu courants, l'hypopyon et l'atteinte du segment postérieur peuvent être présents dans un cas d'uvéite associée au HLA-B27 et peuvent même atteindre les personnes âgées. De plus, il importe d'inclure une évaluation détaillée des segments antérieurs et postérieurs devant toute présentation d'œil rouge douloureux afin d'avoir un profil clinique plus complet. 


\section{Remerciements}

Nous remercions Gerald Abruzzese,

OD, du Syracuse Veteran's Medical

Center, de son aide et de ses conseils.

Ce rapport était présenté en partie au congrès annuel de l'Académie américaine en optométrie $(A A O)$ à Boston le

14 octobre 2011

\section{Références}

1. Rothova A, Suttorp-van Schulten MS, Frits Treffers W, Kijlstra A. Causes and frequency of blindness in patients with intraocular inflammatory disease. $\mathrm{Br} \mathrm{J}$ Ophthalmol 1996;80(4):332-6.

2. McCannel CA, Holland GN, Helm CJ, et al. Causes of uveitis in the general practice of ophthalmology. UCLA Community-Based Uveitis Study Group. Am J Ophthalmol 1996;121(1):35-46.

3. Brewerton DA, Caffrey M, Nicholls A, et al. Acute anterior uveitis and HL-A 27. Lancet 1973;302(7836):994-6.

4. Feltkamp TE. Ophthalmological significance of HLA associated uveitis. Eye (Lond) 1990;4 ( Pt 6):839-44.

5. Wakefield D, Chang JH, Amjadi S, et al. What is new HLA-B27 acute anterior uveitis? Ocul Immunol Inflamm 2011;19(2):139-44.

6. Zaidi AA, Ying GS, Daniel E, et al. Hypopyon in patients with uveitis. Ophthalmology 2010;117(2):366-72.

7. Kase S, Namba K, Horie Y, et al. Repeated exacerbations of ocular inflammation with vitreous hemorrhage in a patient with HLA-B27 associated uveitis. J Med Invest 2007;54(3-4):350-3.

8. Dodds EM, Lowder CY, Meisler DM. Posterior segment inflammation in HLA-B27+ acute anterior uveitis: clinical characteristics. Ocul Immunol Inflamm 1999;7(2):85-92.

9. Rothova A. Comment on 'Posterior segment inflammation in HLA-B27+ acute anterior uveitis: clinical characteristics'. Ocul Immunol Inflamm 2000;8(1):73-5.
10. Tay-Kearney ML, Schwam BL, Lowder $\mathrm{C}$, et al. Clinical features and associated systemic diseases of HLA-B27 uveitis. Am J Ophthalmol 1996;121(1):47-56.

11. Schumacher HR, Jr. The pathogenesis of gout. Cleve Clin J Med 2008;75 Suppl 5:S2-4.

12. Dawson CR, Togni B. Herpès simplex eye infections: clinical manifestations, pathogenesis and management. Surv Ophthalmol 1976;21(2):121-35.

13. Santos C. Herpes simplex uveitis. Bol Asoc Med P R 2004;96(2):71-4, 7-83.

14. Mendes D, Correia M, Barbedo M, et al. Behcet's disease--a contemporary review. J Autoimmun 2009;32(3-4):178-88.

15. Cozier YC, Berman JS, Palmer JR, et al. Sarcoidosis in black women in the United States: data from the Black Women's Health Study. Chest 2011;139(1):144-50.

16. Uyama M. Uveitis in sarcoidosis. Int Ophthalmol Clin 2002;42(1):143-50.

17. Jones NP. Sarcoidosis and uveitis. Ophthalmol Clin North Am 2002;15(3):319-26, vi.

18. Park YH, Han JH, Nam HW. Clinical features of ocular toxoplasmosis in Korean patients. Korean J Parasitol 2011;49(2):167-71.

19. Bornand JE, de Gottrau P. Uveitis: is ocular toxoplasmosis only a clinical diagnosis? Ophthalmologica 1997;211(2):87-9.

20. Thean JH, Hall AJ, Stawell RJ. Uveitis in Herpes zoster ophthalmicus. Clin Experiment Ophthalmol 2001;29(6):406-10.

21. Hong MC, Sheu SJ, Wu TT, Chuang CT. Ocular uveitis as the initial presentation of syphilis. J Chin Med Assoc 2007;70(7):274-80.

22. Reddy S, Cubillan LD, Hovakimyan A, Cunningham ET, Jr. Inflammatory ocular hypertension syndrome (IOHS) in patients with syphilitic uveitis. Br J Ophthalmol 2007;91(12):1610-2.

23. Tucker JD, Li JZ, Robbins GK, et al. Ocular syphilis among HIV-infected patients: a systematic analysis of the literature. Sex Transm Infect 2011;87(1):4-8.

24. Lutchman C, Weisbrod DJ, Schwartz CE. Diagnosis and management of syphilis after unique ocular presentation.
Le Médecin de famille canadien 2011;57(8):896-9.

25. Varma D, Anand S, Reddy AR, et al. Tuberculosis: an under-diagnosed etiological agent in uveitis with an effective treatment. Eye (Lond) 2006;20(9):1068-73.

26. Ramsay A, Lightman S. Hypopyon uveitis. Surv Ophthalmol 2001;46(1):1-18.

27. D'Alessandro LP, Forster DJ, Rao NA. Anterior uveitis and hypopyon. Am J Ophthalmol 1991;112(3):317-21.

28. Nussenblatt RB. Uveitis in Behcet's disease. Int Rev Immunol 1997;14(1):67-79.

29. Jabs DA, Nussenblatt RB, Rosenbaum JT. Standardization of uveitis nomenclature for reporting clinical data. Results of the First International Workshop. Am J Ophthalmol 2005;140(3):509-16.

30. Bloch-Michel E. Opening address: intermediate uveitis. Dev Ophthalmol 1992;23:1-2.

31. Chan SM, Hudson M, Weis E. Anterior and intermediate uveitis cases referred to a tertiary centre in Alberta. Journal canadien d'ophtalmologie 2007;42(6):860-4.

32. Babu BM, Rathinam SR. Intermediate uveitis. Indian J Ophthalmol 2010;58(1):21-7.

33. Zierhut M, Foster CS. Multiple sclerosis, sarcoidosis and other diseases in patients with pars planitis. Dev Ophthalmol 1992;23:41-7.

34. Breeveld J, Rothova A, Kuiper H. Intermediate uveitis and Lyme borreliosis. Br J Ophthalmol 1992;76(3):181-2.

35. Miserocchi E, Waheed NK, Dios E, et al. Visual outcome in herpes simplex virus and varicella zoster virus uveitis: a clinical evaluation and comparison. Ophthalmology 2002;109(8):1532-7.

36. Liesegang TJ. Ocular herpes simplex infection: pathogenesis and current therapy. Mayo Clin Proc 1988;63(11):1092-105.

37. Schacher S, Garweg JG, Russ C, Bohnke M. [Diagnosis of herpetic uveitis and keratouveitis]. Klin Monbl Augenheilkd 1998;212(5):359-62.

38. Rodriguez A, Power WJ, Neves RA, Foster CS. Recurrence rate of 
herpetic uveitis in patients on longterm oral acyclovir. Doc Ophthalmol 1995;90(4):331-40.

39. Liesegang TJ. Herpes simplex virus epidemiology and ocular importance. Cornea 2001;20(1):1-13.

40. Kaye S, Choudhary A. Herpes simplex keratitis. Prog Retin Eye Res 2006;25(4):355-80.

41. Liesegang TJ. Classification of herpes simplex virus keratitis and anterior uveitis. Cornea 1999;18(2):127-43.

42. Kataria RK, Brent LH. Spondyloarthropathies. Am Fam Physician 2004;69(12):2853-60.

43. Rodriguez A, Calonge M, PedrozaSeres M, et al. Referral patterns of uveitis in a tertiary eye care center. Arch Ophthalmol 1996;114(5):593-9.

44. Power WJ, Rodriguez A, PedrozaSeres M, Foster CS. Outcomes in anterior uveitis associated with the HLA-B27 haplotype. Ophthalmology 1998;105(9):1646-51.

45. Rodriguez A, Akova YA, Pedroza-Seres M, Foster CS. Posterior segment ocular manifestations in patients with HLAB27-associated uveitis. Ophthalmology 1994;101(7):1267-74.

46. Smith JR. HLA-B27--associated uveitis. Ophthalmol Clin North Am 2002;15(3):297-307.

47. Lee FF, Foster CS. Pharmacotherapy of uveitis. Expert Opin Pharmacother 2010;11(7):1135-46.

48. Tripathi RC, Parapuram SK, Tripathi BJ, et al. Corticosteroids and glaucoma risk. Drugs Aging 1999;15(6):439-50.

49. Noone T. An overview of steroid use and its potential side-effects. Nurs Times 2006;102(17):24-7.

50. Ferrante P, Ramsey A, Bunce C, Lightman S. Clinical trial to compare efficacy and side-effects of injection of posterior sub-Tenon triamcinolone versus orbital floor methylprednisolone in the management of posterior uveitis. Clin Experiment Ophthalmol 2004;32(6):563-8.

51. Riordan-Eva P, Lightman S. Orbital floor steroid injections in the treatment of uveitis. Eye (Lond) 1994;8 ( Pt 1): 66-9.
52. Antcliff RJ, Spalton DJ, Stanford $\mathrm{MR}$, et al. Intravitreal triamcinolone for uveitic cystoid macular edema: an optical coherence tomography study. Ophthalmology 2001;108(4):765-72.

53. Yalcindag FN, Can E, Ozdemir O. Intravenous methylprednisolone pulse therapy for acute posterior segment uveitis attacks in Behcet's disease. Ann Ophthalmol (Skokie) 2007;39(3):194-7.

54. Wakefield D, McCluskey P, Penny R. Intravenous pulse methylprednisolone therapy in severe inflammatory eye disease. Arch Ophthalmol 1986;104(6):847-51.

55. Okada AA. Immunomodulatory therapy for ocular inflammatory disease: a basic manual and review of the literature. Ocul Immunol Inflamm 2005;13(5): 335-51.
56. Bom S, Zamiri P, Lightman S. Use of methotrexate in the management of sight-threatening uveitis. Ocul Immunol Inflamm 2001;9(1):35-40.

57. Monnet D, Breban M, Hudry C, et al. Ophthalmic findings and frequency of extraocular manifestations in patients with HLA-B27 uveitis: a study of 175 cases. Ophthalmology 2004;111(4): 802-9.

58. Rosenbaum JT. Acute anterior uveitis and spondyloarthropathies. Rheum Dis Clin North Am 1992;18(1):143-51.
NOVA SOUTHEASTERN UNIVERSITY College of Optometry, Ottice of Continuing Education

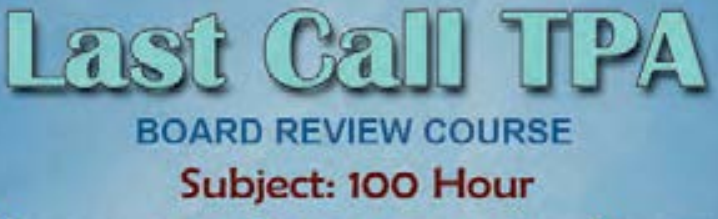

Therapeutic Pharmaceutical Agents Certification Course Dates: July 8 -18, 2012 Hours can be customized
or state/province requirements
or board certification review.

For further information and to register: Web: optometry.nova.edu/ce Tel: (954) 262-4224

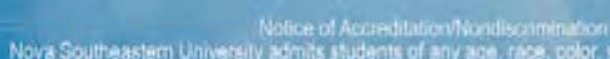

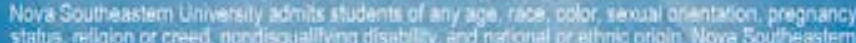

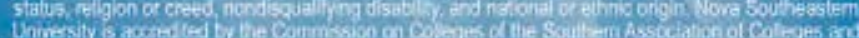

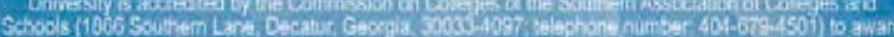

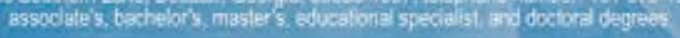

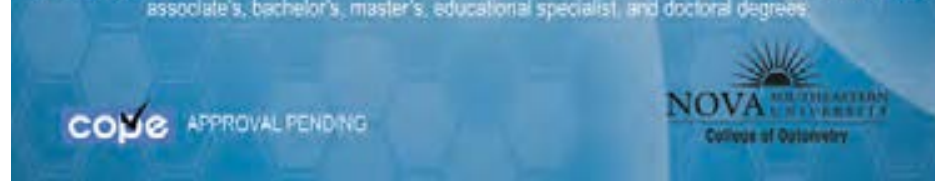

